DOI: 10.21892/978-958-5547-65-0.7

\title{
7. Aplicación de ideas de innovación ambiental: implementación de un sistema de captación de agua por humedad atmosférica en la Región de los Montes de María del departamento de Bolívar
}

\author{
Luis Alberto Díaz González \\ Mitchel Omar Molina Ozuna²
}

Los Montes de María ubicada en la costa caribe colombiana, posee una extensión de $2.677 \mathrm{Km}^{2}$ distribuidos en 15 municipios de los cuales 8 pertenecen a la jurisdicción del departamento de Bolívar y los restantes 7 al departamento de Sucre, siendo conocido el municipio del Carmen de Bolívar como la capital de esta subregión del país.

Los Montes de María, son una subregión rica en flora y fauna, con tierras fértiles aptas para el desarrollo de cultivos agrícolas, y en cuyo territorio existen gran cantidad de cuerpos de agua que posibilitan el desarrollo rural de este lugar, tanto así que las actividades económicas se encuentran representadas en la producción agropecuaria en especial la ganadería bovina y cultivos tradicionales como maíz, arroz, yuca, ñame, plátano, tabaco, café y aguacate, siendo últimamente introducidos a la subregión otros cultivos de tipo empresarial como lo es el ají picante, el cacao y la palma de aceite.

Todo este perfil de los Montes de María, hace considerar que esta subregión tiene todas las condiciones para ser una de las más pujantes en materia de desarrollo rural no solo a nivel de la costa caribe sino del país, sin embargo, factores asociados al abandono estatal que ha sufrido por décadas esta subregión, los problemas de conflicto armado que aún se encuentran arraigados en las mentes de sus pobladores, la desigualdad

1 Ingeniero Químico de la Universidad de Cartagena. Maestría en Desarrollo y Ambiente, en curso, de la Universidad Tecnológica de Bolívar.

2 Ingeniero Químico de la Universidad de Cartagena. Maestría en Desarrollo y Ambiente, en curso, de la Universidad Tecnológica de Bolívar. 
Aplicación de ideas de innovación ambiental: implementación de un sistema de captación de agua por humedad atmosférica en la Región de los Montes de María del departamento de Bolívar

económica entre el empresario que comercializa los productos agrícolas y el campesino que cultiva la tierra, la no inclusión del campesino en la economía de mercado neoliberal impuesta desde los años 90 y la geografía accidentada de gran parte de su territorio, han sido obstáculos para el desarrollo no solo económico sino también humano de los habitantes de esta parte del caribe colombiano.

De acuerdo con todo lo expuesto, los Montes de María se constituyen como una gran dispensa agrícola en la que el campesino el cual es de escasos recursos debe producir la cantidad de alimentos para su subsistencia con los elementos disponibles en la región, que como hemos dicho son abundantes en materia de desarrollo agrícola pero que no están al alcance y disponibilidad de todos, en especial el recurso vital, que es indispensable para una adecuada productividad agrícola pero que lastimosamente por la dinámica del mercado este recurso vital llamado agua está en manos en gran parte de las grandes agroindustrias de onocultivos que se han apoderado poco a poco de las mejores tierras, desplazando así a los cultivos tradicionales y colocando en riesgo la seguridad alimentaria en la zona.

Dentro de este contexto, se visualiza que uno de los grandes problemas que poseen los campesinos para el desarrollo de sus cultivos es el acceso y disponibilidad del agua la cual afecta enormemente los productos de sus cosechas. Conforme a lo estipulado por la Hoja de Ruta para la región de los Montes de María (2014), se pueden establecer dos factores que afectan la productividad agrícola regional: el primer factor hace referencia a la deficiente infraestructura de riego; "la irrigación de manera general para la productividad de los cultivos es precaria y la poca realizada se hace por grandes y medianos productores y el pequeño productor solo lo hace de manera artesanal y de manera costosa.

El territorio cuenta con una infraestructura de riego conformada por 32 minidistritos de riego, del cual se benefician $955 \mathrm{Ha}$, especialmente los monocultivos; 15 de estos se encuentran en Bolívar y 17 en Sucre, pero 25 de estos están inactivos" (Hoja de Ruta para la región de los Montes de María, 2014, p: 48). El segundo factor consiste en las perdidas de cosechas por fenómenos climáticos; "la región presenta problemas de sedimentación e inundaciones en invierno, porque la capacidad de drenaje es muy baja. 
En épocas de sequía hay escasez de aguas subterráneas y es así como ante los últimos descensos continuos en los volúmenes de agua lluvia debido al cambio climático o fenómeno del niño desde el año 2013 viene agravando la situación y ha provocado que en los últimos años se hayan presentado, una sequía de los jagüeyes para consumo humano y animal" (Hoja de Ruta para la región de los Montes de María, 2014, p: 48).

Estos dos factores, limitan la productividad agrícola en una subregión como lo es la de los Montes de María, convirtiéndose en grandes obstáculos para el desarrollo rural de esta zona del caribe colombiano. El primer factor deja ver que la carencia de infraestructura de riego hace insostenible los cultivos agrícolas que se desarrollan por parte de los campesinos en especial los pequeños productores, ya que no tienen un acceso a la red hídrica de distritos de riego ocasionado por el poco mantenimiento que se le hace a los mismos para el aprovechamiento de todos los productores y por la disposición de estos a alimentar los monocultivos que año tras año aumentan sus hectáreas en lo Montes de María y dejando de lado la productividad local tradicional que por décadas ha caracterizado a esta subregión del país.

De acuerdo a Ríos et. al (2012), "El negocio de la palma, lo integran cerca de 490 pequeños y medianos productores, de los cuales el 95\% cultiva en promedio 7.5 hectáreas, mientras que solo cuatro productoras cultivan áreas con un promedio de 400 hectáreas. Esta situación es extremadamente paradójica teniendo en cuenta los enormes atrasos en la infraestructura hídrica de la región y especialmente del departamento de Bolívar, ya que el mayor distrito de riego es el de María la Baja con 11.873 hectáreas, mientras que el resto son 24 minidistritos con menos de 10 hectáreas de espejo de agua, 20 de las cuales están actualmente inactivas, lo que genera riesgo de sostenibilidad productiva de las familias campesinas que requieren del recurso hídrico para el cultivo" (p: 41).

En consecuencia, como el campesino no tiene acceso a estos distritos de riego, debe planificar sus cultivos conforme al régimen de lluvias que se presenta en la zona, lluvias que tienen una duración aproximada de tres meses para abastecer los cultivos del agua necesaria para su producción. Es en este sentido, cuando cobra fuerza el segundo factor que se ha mencionado, donde el clima juega un papel fundamental en la productividad agrícola de los Montes de María. 
Aplicación de ideas de innovación ambiental: implementación de un sistema de captación de agua por humedad atmosférica en la Región de los Montes de María del departamento de Bolívar

El cambio climático ha ocasionado que las sequias sean más intensas que en décadas anteriores, lo que provoca que las aguas superficiales y subterráneas escaseen para la actividad agrícola y colocando en riesgo la canasta familiar de los campesinos que subsisten de sus propios cultivos, población que es altamente mayoritaria en los Montes de María. Por esta situación, desde el año 2013, se han generado muchas pérdidas de cultivos de productos tradicionales de la zona, colocando en riesgo la seguridad alimentaria en primer lugar de los campesinos que subsisten de sus propias cosechas.

En este contexto, se puede decir que el problema principal para un adecuado desarrollo agrícola en especial de los campesinos más vulnerables es el limitado acceso a las fuentes de agua para el regado de sus cultivos. Hasta hoy día en los Montes de María, las fuentes de agua de abastecimiento tanto para consumo humano como para su uso en sistemas de regadío de cultivos provienen de fuentes superficiales como ríos, lagunas, jagüeyes, arroyos, y fuentes subterráneas como lo son los conocidos pozos de agua, ojos de agua, etc.

Debido a la intensa humedad que existe en esta zona del país que ronda alrededor de los 90 a 95\%, la evapotranspiración provoca que grandes cantidades de agua provenientes de las fuentes ya mencionadas se transfieran del estado líquido en el que se encuentran comúnmente, al estado gaseoso combinándose así con las masas de aire que componen la atmosfera. En este sentido, el aire al contener agua en forma gaseosa, se convierte en una fuente del recurso vital que para los pobladores de esta subregión ha pasado desapercibido a lo largo de los años.

Por tal motivo, el aire se constituye en un medio portador de agua que mediante un proceso adecuado puede obtenerse en forma líquida para que sea utilizada tanto para el consumo humano como para su uso en el regadío de cultivos agrícolas, bajo la premisa de que el aire es un bien público y por lo tanto nos pertenece a todos y de igual forma todos tenemos acceso a él.

La idea de contemplar la posibilidad de obtener agua a partir del aire en una subregión donde la humedad del aire indica la presencia de gran cantidad de agua en este medio, se constituye en una alternativa de solución para mitigar los problemas de captación del preciado líquido para su uso en las actividades agrícolas en épocas distintas a las de lluvias o 
mejor dicho, con la captación de agua a partir del aire se pueden planificar cultivos en épocas de intenso verano con el objetivo de que los pequeños campesinos que no tienen acceso a los sistemas de regadío o distritos de agua, puedan ser productivos a lo largo del año, utilizando una tecnología que devuelve al sustrato terrestre el agua que por evapotranspiración se ha ido a la atmosfera.

En este sentido, se plantea la puesta en marcha de un sistema tecnológico amigable con el medio ambiente que utiliza los principios de condensación del agua y de la generación de energía por acción de la luz solar, para la captación, almacenamiento y distribución en sistemas de regadío para cultivo agrícolas tradicionales en beneficio del pequeño campesino, favoreciendo el desarrollo económico, humano y rural de estas comunidades de los Montes de María.

La captación de agua a partir del aire es una idea que se ha concretado en otros países como Perú y Chile sobre todo en sus zonas desérticas donde existen elevados valores de humedad del aire que permiten la condensación del agua, utilizando para esto un sistema de lluvia horizontal o de atrapanieblas. "Los orígenes de los atrapanieblas se remontan a Chile en los años 60, donde una crisis hídrica en la ciudad de Antofagasta (1956) inspira un grupo de investigadores encabezados por el profesor Carlos Espinosa, para aprovechar el potencial de la niebla, Camanchacal, del desierto de Atacama, que se forma de la humedad del océano pacífico.

Tras patentar la invención, es donada a la Universidad Católica de Chile, y se fomentó su difusión a través de la UNESCO, para favorecer a las comunidades más vulnerables. Después de algunas pruebas, un sencillo sistema conformado por una malla atada a un par de postes, logra captar el agua que viajaba como vapor. El agua colectada pudo ser de consumo humano, logrando generar una oferta que permitió el riego de cultivos". (Mendoza, Castañeda, 2014, p: 16).

La aplicación de esta tecnología se expandió a varios países de América Latina, siendo Colombia uno de los países donde ha tenido éxito el aprovechamiento de la neblina del aire para la captación de agua para el regado de pequeños cultivos, como es el caso del corregimiento de Frisoles en el municipio de Guadalajara de Buga en el departamento del Valle del Cauca, donde la Corporación del Valle del Cauca, CVC, entidad ambiental, 
Aplicación de ideas de innovación ambiental: implementación de un sistema de captación de agua por humedad atmosférica en la Región de los Montes de María del departamento de Bolívar

implementó un sistema de captación de agua por medio de una tela denominada atrapanieblas para su uso en el regado de pequeños cultivos que desarrollan los campesinos de la región.

Ahora bien, se ha visto que el factor común para la obtención de agua es la presencia de niebla en la zona, fenómeno que aunque se presenta en la subregión de los Montes de María, no es muy abundante en comparación a lo que si representa la humedad del aire donde por sus valores es posible obtener el agua contenida en las masas de aire a través de un sistema similar en el que se utiliza el principio de condensación del agua con la que funciona la malla atrapanieblas, adaptada a un sistema de condensación diseñado conforme a las características del aire de esta zona del país, en el que choquen las masas de aire que contienen el agua que se encuentra a una temperatura superior a la superficie de contacto del condensador que se encuentra a una temperatura mucho más baja, y que permite por intercambio de calor llevar el agua que se encuentra en estado gaseoso al estado líquido para su almacenamiento y posterior distribución en sistemas de regadío de cultivos tradicionales en la subregión de los Montes de María.

De acuerdo a Bautista - Olivas et al (2011), "La humedad atmosférica es una fuente alternativa de agua potable en las zonas áridas y semiáridas, y se puede complementar con las fuentes tradicionales en los asentamientos agrícolas y en los sistemas de abastecimiento urbano de agua en estas regiones, como ocurre en Chile, Perú, Namibia e Islas Canarias, donde se utiliza como una fuente de agua potable". (p: 294).

En este sentido, la aplicación de tecnologías de captura de agua a partir del aire es una alternativa viable para la sostenibilidad agrícola de los Montes de María, y representa una forma de desarrollo rural nunca antes puesta en marcha en esta zona del país, siendo así esto un mecanismo de innovación en materia ambiental, ya que se genera un impacto favorable al ambiente en el sentido de que se aprovecha un recurso presente en el aire a partir del funcionamiento de un condensador que se alimenta de energía solar, adaptando los sistemas de captura de agua del aire propuestos en otros países, a las condiciones climatológicas de los Montes de María, para lo cual se contempla un reajuste en los diseños de implementación y funcionamiento de dicha tecnología que permite optimizar el proceso de deshumidificacion del aire que se experimenta al obtener agua de este medio. 
Teniendo en cuenta lo establecido por Castro (2011), que señala que "Dentro del campo del Diseño ambiental, el papel que juega la investigación científica y el desarrollo tecnológico, no sólo desde las prácticas, sino desde la formación del pensamiento científico, de la indagación permanente, de la sistematización, de la creación e invención, que se retroalimenta de la cultura y las artes, que da lugar, en últimas a la INNOVACIÓN" (p:271), la investigación aplicada se convierte en un mecanismo de desarrollo para las regiones, pues presenta soluciones a las problemáticas que se identifican en un lugar determinado, que en este caso es la carencia de un adecuado acceso y distribución del agua para el desarrollo de cultivos agrícolas en la subregión de los Montes de María.

Álvarez (2004) plantea que una innovación ambiental debe cumplir con tres requisitos: ventaja relativa, compatibilidad y complejidad. El primer requisito se define como "el grado en el cual una innovación se percibe mejor que la idea que supera y se puede expresar como un beneficio económico, prestigio social u otros beneficios" (Álvarez, 2004, p: 69).

Llevando esta característica al contexto de captación de agua a partir de la humedad del aire, la ventaja relativa que presenta esta idea radica en que la obtención de agua es posible a partir de un fenómeno diferente al de la niebla, lo cual supera la idea de innovación que se tiene al respecto de la obtención de agua y que se ha aplicado en distintos lugares. Esta concepción de superación de la idea actual que se tiene, supone un principio de innovación en la tecnología de captación de agua a partir de otro fenómeno que en este caso es la humedad del aire.

Ahora bien, la implementación de esta tecnología en los Montes de María, otorgaría más que un prestigio social, el aprovechamiento de forma sostenible de los recursos hídricos para el desarrollo agrícola de la región, teniendo en cuenta que a partir del uso de la humedad del aire como fuente de agua, en esta zona del país no se ha realizado ninguna puesta en marcha de alguna tecnología que permita realizar esta función.

La implementación de esta tecnología traería consigo muchos beneficios a los pequeños campesinos ya que tendría un medio para acceder al agua que por la distribución y manejo que el mercado y las empresas de monocultivos les han dado a los distritos y minidistritos de riego, puedan 
Aplicación de ideas de innovación ambiental: implementación de un sistema de captación de agua por humedad atmosférica en la Región de los Montes de María del departamento de Bolívar

aumentar su productividad agrícola, extendiendo sus periodos de cultivos incluso en épocas de baja precipitación o intenso verano.

El segundo requisito, la compatibilidad, "es el grado en el cual una innovación se percibe consistente con los valores existentes" (Álvarez, 2004, p: 70). Dentro de este requisito es de anotar que la implementación de una tecnología como la de captación del agua a partir de la humedad del aire es aplicable a la zona de los Montes de María debido a que los valores de humedad del aire que posee la zona, son los ideales para la puesta en marcha del modelo tecnológico que permita dicha captación del agua, y que a la vez permite construir nueva información relacionada con las condiciones climatológicas necesarias para la expansión de la tecnología por todo el caribe colombiano, bajo la premisa de que la humedad del aire en esta región del país maneja rangos homogéneos entre los 75\% y 95\%, lo que la hace aplicable a otras zonas del Caribe. La aplicación de esta tecnología en los Montes de María, sentaría las bases para la construcción de cartas psicométricas en esta subregión con la finalidad de conocer los periodos de máxima y mínima obtención de agua a partir de la humedad del aire, que acompañado con el conocimiento tradicional de los campesinos de la zona, se pueden establecer parámetros de diseño de sistemas de regadío para los cultivos que permitan la productividad de los mismos a lo largo del año.

El tercer requisito, la complejidad, "es el grado en que una innovación se percibe como difícil de entender y usar, mientras más compleja se perciba menos aceptación tendrá" (Álvarez, 2004, p: 70).

En este sentido, la aplicación de esta tecnología no representa ninguna complejidad en su puesta en marcha ya que simplemente es un sistema que capta agua, la cual se encuentra en el aire a una temperatura mayor a la de la superficie de contacto del condensador, lo que provoca que el agua pase del estado gaseoso al estado líquido, y se almacene en tanques para su distribución a los sistemas de regadío de los cultivos.

Con esta información se pueden destacar tres partes fundamentales del sistema de captación de agua: condensador, tanque de almacenamiento y tubería de distribución. Sin embargo, el sistema para su aplicabilidad en el contexto en el que se ejecuta el cual son los Montes de María, debe ser de fácil manejo por parte de los campesinos, ya que en su manejo es donde 
se encuentra la aceptación de la tecnología para la implementación en los cultivos que cosechan.

La innovación ambiental es parte fundamental de un adecuado desarrollo sostenible tal y como lo afirma Bárcena (2001), "el desarrollo sostenible debe convertirse en el pilar de la transición de la modernidad hacia una posmodernidad caracterizada por cambios en los modos de producción y consumo acompañados de la innovación tecnológica con fundamentos de sostenibilidad ambiental, por lograr la competitividad económica a partir de la excelencia ambiental, por la valoración de la diversidad natural y cultural, por el fortalecimiento de lo local y por la democracia con ciudadanía potenciada por los avances en la comunicación y los sistemas de información". (p: 91).

Esta premisa potencializa la necesidad de hacer prácticas de innovación ambiental que permitan mitigar las consecuencias del cambio climático, con la participación activa de todos los actores de la sociedad que para el caso de los Montes de María serian en primer lugar los campesinos que cultivan la tierra, las administraciones municipales de las entidades territoriales que conforman esta subregión del caribe colombiano, la administración regional representada por los departamentos que incluyen esta zona dentro de su territorio, la administración nacional representada por las entidades involucradas en el desarrollo tecnológico ambiental sostenible y la academia representada en Universidades que poseen el conocimiento técnico y científico para la construcción de ideas innovadoras como la planteada para la captación de agua a partir de la humedad del aire. En definitiva, la implementación de las ideas de innovación ambiental depende en gran medida de una adecuada gobernanza que permita la participación activa de todos estos actores mencionados.

Sin embargo, la innovación ambiental, además de ser una estrategia para promover un desarrollo sostenible, posee muchos obstáculos siendo el principal la financiación de estas tecnologías. "Aunque se ha producido cierto aumento en el gasto en ciencia, tecnología y entrenamiento (en algunos países mucho más que en otros), esta inversión sigue siendo insuficiente en la mayoría de las circunstancias para poder lograr una 'masa crítica' de capital humano, infraestructura y recursos destinados a la I\&D o entrenamiento. Esta escasez general de inversión de recursos persiste y tiene implicaciones para la producción en investigación y tecnología" 
Aplicación de ideas de innovación ambiental: implementación de un sistema de captación de agua por humedad atmosférica en la Región de los Montes de María del departamento de Bolívar

(Arond et. al, 2011, p: 46). La aplicación de esta tecnología y el avance en su investigación para su optimización, requiere de recursos financieros y humanos que permitan colocar en prácticas estas ideas de innovación ambiental.

Las políticas regionales contemplan el desarrollo sostenible como un mecanismo de crecimiento económico, pero quizás los muchos requisitos que se exigen para financiar un estudio de investigación que permita colocar en marcha una tecnología ambiental innovadora, puede provocar que innovaciones ambientales como la propuesta en el presente escrito no se lleven a cabo o no se trabajen a profundidad. Es posible que los recursos económicos existan, pero también debe haber un mecanismo flexible y de calidad que incentive el desarrollo de ideas innovadoras para concretarlas en proyectos que permitan el desarrollo sostenible de la subregión de los Montes de María.

Según Porter \& Linde (1999), "Innovar para cumplir con las regulaciones pueden traer compensaciones: el uso de mejores insumos, la creación de mejores productos, o la mejora de los rendimientos del producto". En este sentido, la innovación ambiental constituye un mecanismo de mejora de los sistemas de producción que se realizan en la subregión de los Montes de María, pues permite que durante las épocas de poca precipitación y/o intenso verano, los campesinos puedan seguir con sus actividades de cultivar la tierra pues se estaría garantizando el acceso al preciado líquido, que debido a las condiciones actuales que se evidencian en los Montes de María con respecto a la disponibilidad de agua para su uso en regadíos, son muy pocas, lo cual le otorgaría al campesino en especial al pequeño productor, mantener sus cultivos para obtener de ellos los productos necesarios para su canasta familiar y la comercialización de los mismos.

La implementación de esta tecnología de captación de agua a partir de la humedad del aire en los Montes de María, representaría para muchos campesinos una oportunidad de vincularse al mercado para así hacer sus cosechas competitivas, pues tendrían a disposición el recurso vital necesario para el desarrollo de sus cultivos. Esta situación, generaría un equilibrio en la dinámica comercial de la región, ya que productores que solo cultivaban conforme a las épocas de lluvia, ya lo pueden hacer incluso durante todo el año, pues tienen a su disposición una tecnología amigable con el medio 
ambiente que les permite captar agua de una fuente como es el aire, que en una región como los Montes de María, jamás ha sido explotada.

Si bien es cierto que el agua se puede obtener a partir de la humedad del aire, la calidad del aire en las zonas rurales muestra valores muy positivos en comparación a las ciudades, ya que el aire rural presenta mucho menos contaminación, lo que permite intuir que la calidad del agua obtenida es muy buena y que no necesita de un tratamiento exhaustivo para el consumo humano, en comparación a los tratamientos que se realizan a las aguas superficiales para su consumo. De acuerdo a este panorama, si el agua obtenida por captación de la humedad del aire es consumida, los costos de tratamiento para su potabilización son mínimos en comparación con la del tratamiento de aguas provenientes de fuentes convencionales.

La temática de implementación de tecnologías ambientales innovadoras, abre las puertas para el desarrollo sostenible de subregiones como la de los Montes de María, la cual tiene todas las condiciones para ser una región pujante en materia de agricultura. El acceso al agua ha sido el principal problema para el rendimiento al máximo de sus cosechas y teniendo en cuenta el principio de Lavoisier de conservación de la masa, el agua en los Montes de María es constante, donde la liquida, aquella que está presente en espejos de agua ya sea de ríos, lagunas, jagüeyes, etc. y otras subterráneas como pozos, no estén disponibles para su acceso al pequeño campesino por su apropiación en especial de los grandes monocultivos (palma), el agua que no se ve, que se encuentra en forma gaseosa, es una alternativa para que estos grupos de pequeños productores puedan abastecerse para el regadío de sus cultivos. Es lógico que de implementarse esta tecnología en los Montes de María, se sentarían las bases para el inicio del desarrollo rural de esta zona del país.

Las innovaciones son un excelente paso para prevenir y mitigar los efectos del cambio climático, siempre y cuando sean amigables con el ambiente, lo cual abre la puerta a que se desarrollen criterios legislativos que permitan la explotación de fuentes renovables para consolidar un adecuado desarrollo económico y humano en subregiones como la de los Montes de María. 
Aplicación de ideas de innovación ambiental: implementación de un sistema de captación de agua por humedad atmosférica en la Región de los Montes de María del departamento de Bolívar

\section{Referencias}

Álvarez, L. (2004). Innovación tecnológica y contaminación ambiental. Revista Economía informa 330: 65 - 72.

Arond, E., Rodríguez, L., Arza, V., Herrera, F., Sánchez, M. (2011). Innovación, sustentabilidad, desarrollo e inclusión social: lecciones desde América Latina, STEPS Working paper 48, Brighton: STEPS Centre.

Bárcena, A. (2000). Principales desafíos ambientales en América Latina y el Caribe. En: Actas del seminario internacional "Experiencia latinoamericana en manejo ambiental" CEPAL: 91 - 98.

Bautista-Olivas, A., Tovar - Salinas, J., Palacios - Vélez, O., Mancilla Villa, O. (2011). La humedad atmosférica como fuente opcional de agua para uso doméstico. Revista Agrociencia 45: 293 - 301.

Castro, M. (2011). Ciencia e innovación tecnológica en el diseño ambiental. Revista arq.urb 6: $244-272$.

De los Ríos, E., Becerra, C., Oyaga, F. (2012). Montes de María. Entre la consolidación del territorio y el acaparamiento de tierras. Bogotá: Publicaciones ILSA.

Mendoza, B., Castañeda, F. (2014). Criterios Metodológicos para la definición del sistema de captación de aguas con base en lluvia horizontal. Bogotá: Universidad Católica de Colombia.

Porter, M. E., \& Linde, C. (1999). Green and competitive: ending the stalemate. Journal of Business Administration and Policy Analysis, 215. 